\title{
The dynamical response to the node defect in thermally activated remagnetization of magnetic dot array
}

\author{
P. Baláž, D. Horváth, M. Gmitra \\ 'Department of Theoretical Physics and Astrophysics, Faculty of Science, P.J.Šafárik University, Park Angelinum 9 , 04101 Košice, Slovak \\ Republic
}

\section{Abstract}

The influence of nonmagnetic central node defect on dynamical properties of regular square-shaped $5 \times 5$ segment of magnetic dot array under the thermal activation is investigated via computer simulations. Using stochastic Landau-Lifshitz-Gilbert equation we simulate hysteresis and relaxation processes. The remarkable quantitative and qualitative differences between magnetic dot arrays with nonmagnetic central node defect and magnetic dot arrays without defects have been found.

Key words: magnetic dot arrays, thermal activation, stochastic Landau-Lifshitz-Gilbert equation, magnetic hysteresis, magnetic relaxation PACS: 61.80.Az, 74.25.Fy, 74.72.Bk, 02.70.Bf

\section{Introduction}

The term Magnetic dot array (MDA) [1,2,3] refers to the family of nano-scaled monolayer structures consisting of the identical magnetic nanoparticles, called dots, which are periodically ordered on a non-magnetic substrate. MDA concept is compelling, partly because of qualitatively new properties, that essentially differ from those of the bulk materials. Typical for MDA physics is an intricate collective behavior. Its understanding may be valuable for condensed matter physics, material science and nanoscience. The special properties of MDA systems follow from the interplay between intra-dot and inter-dot interactions as well as from the interplay of anomalously large surface compared to the bulk magnetic energy contributions $[4,5]$. The MDA properties are already utilized in technological applications concerning magnetic field sensors $[6,7,8]$ and reading heads of magnetic-disk data-storage devices [9].

The technology of fabrication of MDAs $[10,11,12,13]$ has been perfected to an excellent degree in the past few years. In practice, however, an occurrence of technological defects and local irregularities has still a great influence on all of the magnetic properties [14]. In this paper we study how the imperfection in form of the single-dot vacancy affects remagnetization of MDA under the assistance of thermal activation.

In the series of papers $[15,16,17,18,19]$ the authors focus on remagnetization of small segments of arrays by simulat- ing elementary models where dots are treated as interacting point dipoles. In Ref.[20] we draw the effect of uniaxial anisotropy induced by the eccentrically placed node defect. Later [21] we have investigated how the central defect affects the quasi-static zero-temperature remagnetization of MDA. From this study we know that: (i) the square lattice seems to be more sensitive to defect occurrence than the triangular one; (ii) zero-temperature differences of hysteresis loops are not very pronounced despite of the remarkable changes in the local arrangement of dots. In addition, the study has opened question of defect influence on the relaxation modes.

In the present study the effect of nonmagnetic centralnode defect, in further referred as defect only, is reconsidered. However, the additional realistic factor included here is the thermal activation. In such case the statistical treatment of results is necessary. Therefore, in order to discern defect consequences, two distinct MDA arrangements -defect-free (DF) and defect-including (DI) are compared.

\section{Model}

Because the statistical simulations need computational effort we preferred use of elementary model where each dot is described by the point magnetic dipole. This simplification is justified for monodomain isotropic nearly spherical ferromagnetic particles separated by a sufficient lattice spacing several times exceeding a dot diameter. Addition- 
ally, we focus on the small MDA samples where dots are placed on square $L \times L$ lattice. The magnetic state of $i$-th dot is described by the effective rescaled $3 \mathrm{~d}$ magnetic moment $\mathbf{m}_{i}$ normalized as $\left|\mathbf{m}_{i}\right|=1$. The inter-dot interactions are assumed to be dipolar and described by the effective field

$$
\mathbf{h}_{i}^{\text {dip }}=-\sum_{j=0, j \neq i}^{L \times L} \frac{\mathbf{m}_{j} r_{i j}^{2}-3 \mathbf{r}_{i j}\left(\mathbf{m}_{j} \cdot \mathbf{r}_{i j}\right)}{r_{i j}^{5}},
$$

where $\mathbf{r}_{i j}$ is the distance between $i$-th and $j$-th dot in lattice-spacing units $a$. The field is measured in the $H_{0}=$ $V M_{\mathrm{s}}\left(4 \pi a^{3}\right)^{-1}$ units including the dot volume $V$ and saturated magnetization $M_{\mathrm{s}}$. To study the DI arrangement we assume that defect is represented by the zero magnetic moment. The dynamics of magnetic moments is described by the stochastic Landau-Lifshitz-Gilbert equation [22]

$$
\frac{\mathrm{d} \mathbf{m}_{i}}{\mathrm{~d} \tau}=-\mathbf{m}_{i} \times \mathbf{h}_{i}^{\text {eff }}-\alpha \mathbf{m}_{i} \times\left(\mathbf{m}_{i} \times \mathbf{h}_{i}^{\text {eff }}\right),
$$

where $\alpha$ is the dimensionless damping parameter, $\tau$ is the time in $t_{0}=4 \pi a^{3}\left[\gamma\left(1+\alpha^{2}\right) V M_{\mathrm{s}}\right]^{-1}$ units and $\gamma$ is the gyromagnetic ratio. The effective field $\mathbf{h}_{i}^{\text {eff }}$ includes the dipolar field $\mathbf{h}_{i}^{\text {dip }}$, the external field $\mathbf{h}^{\text {ext }}$, and the Langevin thermal field $\mathbf{h}_{i}^{\text {th }}$. Finally $\mathbf{h}_{i}^{\text {eff }}=\mathbf{h}_{i}^{\text {dip }}+\mathbf{h}^{\text {ext }}+\mathbf{h}_{i}^{\text {th }}$. The random thermal field $\mathbf{h}_{i}^{\text {th }}$ is defined by averages [23]

$$
\begin{aligned}
\left\langle h_{i, \xi}^{\mathrm{th}}(\tau)\right\rangle & =0, \\
\left\langle h_{i, \xi}^{\mathrm{th}}(\tau) h_{j, \eta}^{\mathrm{th}}\left(\tau^{\prime}\right)\right\rangle & =2 D \delta_{i j} \delta_{\xi \eta} \delta\left(\tau-\tau^{\prime}\right),
\end{aligned}
$$

where $\xi, \eta \in\{x, y, z\}$ and $i, j$ are the site indexes; $D$ is the noise amplitude. According to fluctuation-dissipation relation [22,23], the factor $D$ is linked to the temperature $T$

$$
D=\frac{\alpha}{1+\alpha^{2}} \frac{T}{T_{0}}, \quad T_{0}=\frac{\mu_{0} V^{2} M_{\mathrm{s}}^{2}}{4 \pi k_{\mathrm{B}} a^{3}},
$$

where $T_{0}$ is the characteristic temperature scale.

\section{Hysteresis}

In all of our numerical experiments we set $L=5$ for MDA. The main empirical argument supporting our choice $\alpha=0.1$ is that ferrite nanoparticles have the mean value of $\alpha$ of such order [24]. Let us first study the remagnetization in DF and DI systems in the time varying external magnetic field applied in parallel to one of the main MDA axes $\mathbf{h}^{\text {ext }}(\tau)=\left(h_{\mathrm{x}}^{\text {ext }}(\tau), 0,0\right)$. For simulated geometry similarly as in the hypothetical experimental setup the quantity of interest is the magnetization projection $M_{\mathrm{x}}=$ $\frac{1}{L^{2}} \sum_{i=1}^{L^{2}} \mathbf{m}_{i} \cdot \mathbf{e}_{\mathrm{x}}$. The component $h_{\mathrm{x}}^{\mathrm{ext}}(\tau)$ has been cycled within the bounds $-h_{\max }<h_{\mathrm{x}}^{\operatorname{ext}}(\tau)<h_{\max }$. The simulation starts from the nearly saturated state with $M_{\mathrm{x}} \simeq$ 1 at $h_{\mathrm{x}}^{\text {ext }}=h_{\max }$, where $h_{\max }$ is the bound chosen to keep the system of moments saturated. In the remagnetization regime each time-integration step $\Delta \tau=10^{-2}$ is accompanied by the unique change of the external field $\Delta h_{\mathrm{x}}^{\text {ext }}= \pm 10^{-6}$. Both quantities define the sweeping rate
$v_{\mathrm{h}}=\left(\left|\Delta h^{\mathrm{ext}}\right| / \Delta \tau\right) v_{0}=10^{-4} v_{0}$, where $v_{0}=H_{0} / t_{0}$. We observed that numerical results depend on the sweeping rate.

Numerical integration of Eq. (2) has been performed using stochastic predictor-corrector Heun scheme [22]. This choice is justified by the fact that in general, the statistical error of the scheme can be made arbitrarily small by averaging over the number of stochastic paths [25]. We tested numerical stability of scheme for two different integration steps. In order to fix $v_{\mathrm{h}}$, both $\Delta h_{\mathrm{x}}$ and $\Delta \tau$ have been rescaled by $1 / 2$. The test confirmed the invariance of statistical results with respect to the rescaling. Under the conditions of thermal activation we recorded and treated assembly of 600 independent loops. The treatment assumes averaging of the magnetization data conditioned by $\Delta h_{\mathrm{x}}^{\mathrm{ext}}<$ 0 and $\Delta h_{\mathrm{x}}^{\text {ext }}>0$, respectively. In Fig.1 we show averaged loops constructed for $T=0.01 T_{0}, 0.1 T_{0}$ and $0.5 T_{0}$. We see that loops differ for DI MDA and DF MDA variants. The difference confirms some anomalous impact of the local assymetry of the couplings broken by defect. Clearly, by increasing the temperature the hysteresis vanishes due to reduction of the impact of irreversible processes due to configurations separated by energy barriers. When assume stan-
DF
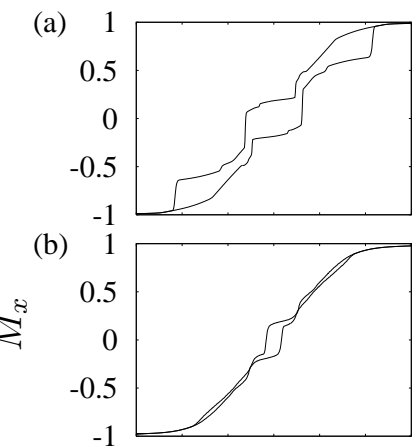

(c)

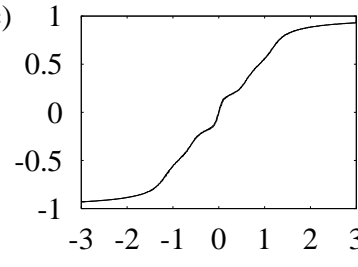

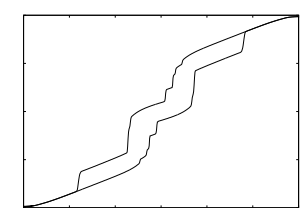
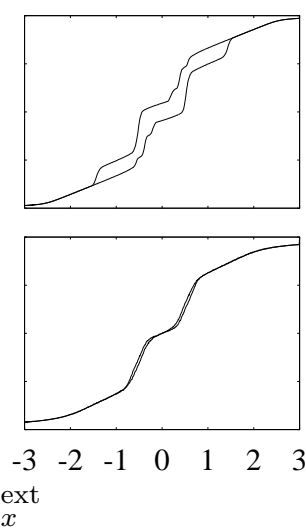

Fig. 1. The averaged hysteresis loops obtained for DF (left panel) and DI (right panel) MDAs at temperatures (a) $T=0.01 T_{0}$, (b) $T=0.1 T_{0}$, (c) $T=0.5 T_{0}$.

dardly that the amount of free energy responsible for the irreversible processes is proportional to the area $A$ of hysteresis loop. According Fig.2 the thermally induced hysteresis reveals maximum of the ratio $A_{\mathrm{DI}} / A_{\mathrm{DF}}$ at $T=0.25 T_{0}$. It is clear that for sufficiently high temperatures the differences between DI and DF systems vanish. Since the remagnetization process of MDAs is very inhomogeneous we analyzed the local mean magnetic hysteresis for nearest neighborhood of central node, corners and middle nodes of MDA edges. The local loops differ among each other. From their comparison it follows that defect affects not only hysteresis 


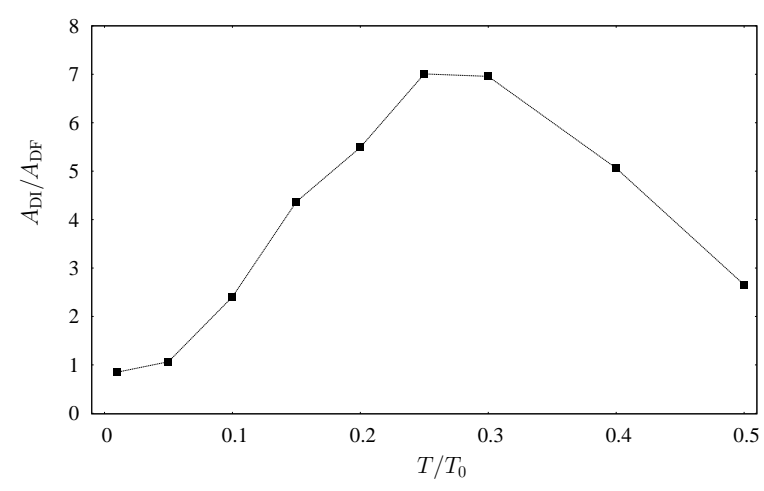

Fig. 2. The ratio of the areas of DI and DF hysteresis loops as a function of the temperature.

of its nearest neighbor dots, but also hysteresis at corners and edges, that clearly follows from the long-range nature of magnetostatic couplings.

One can expect that averaging eliminates some kind of the relevant defect-sensitive information. Therefore, it seems valuable to pay attention to the fluctuations around the averaged loops. To do this we analyzed the noise formed by returns $\Delta M_{\mathrm{x}}=M_{\mathrm{x}}(\tau+100 \Delta \tau)-M_{\mathrm{x}}(\tau)$ accumulated during reversals. Also this data have been treated separately for $\Delta h_{\mathrm{x}}^{\text {ext }}>0$ and $\Delta h_{\mathrm{x}}^{\text {ext }}<0$ sweeps. Evidently, the probability density functions of returns exhibit defect sensitivity. They has been quantitatively characterized by the parameters of leptocurticity $\left\langle\Delta M_{\mathrm{x}}^{4}\right\rangle / 3\left\langle\Delta M_{\mathrm{x}}^{2}\right\rangle^{2}$ and skewness $\left\langle\left|\Delta M_{\mathrm{x}}\right|^{3}\right\rangle /\left\langle\left|\Delta M_{\mathrm{x}}\right|\right\rangle^{3}$. Their numerical values are listed in Table.1. The values clearly indicate that magnetization

\begin{tabular}{ccccc}
\hline & \multicolumn{2}{c}{ leptocurticity } & \multicolumn{2}{c}{ skewness } \\
$T / T_{0}$ & DF & DI & DF & DI \\
\hline \hline 0.01 & 66.52 & 41.31 & 2.28 & 0.81 \\
0.10 & 4.41 & 6.91 & 0.05 & 0.06 \\
0.50 & 1.93 & 1.93 & 0.04 & 0.03 \\
\hline
\end{tabular}

Table 1

Statistical characteristics of the magnetization returns. The leptocurticity and skewness obtained from fluctuations of hysteresis at different temperatures.

noise is strongly non-gaussian. We see that temperature affects the characteristics in common manner, they decrease. However, the remarkable differences between DF and DI systems exist again.

The reversal magnetization paths uncovered complex collective inner behavior of dot moments. In addition the thermal fluctuations cause that single remagnetization events qualitatively differ between each other. We observed that intervals with smoothly varying magnetization, where the quasi-coherent rotation of moments prevails, are broken by the irreversible jumps. To understand redundant but essential attributes of the reversal statistics we exploited the abilities of artificial neural networks. The most efficient for our purposes seems to be the usage of unsupervised self-organizing maps (SOM) $[26,27]$ that allow us to extract several representative paths from the largely redundant assembly. In Fig. 3 we show the representative paths $\left(T=0.01 T_{0}\right)$ extracted by SOM. For certain inner parameters of SOM the classification yields two representative groups of loops (classification is done for DF and DI cases separately). The quantitative output of this analysis is that the averaged loops from Fig. 1(a) can be understood as a mixture of $89 \%$ of (a1) constituent with small addition of $11 \%$ of (a2) in DF case, and $65 \%$ of (b1) with $35 \%$ contribution of (b2) in DI case. This classification explains why the patterns (a1), (b1) are much more similar to the averaged loops than less probable types (a2), (b2).

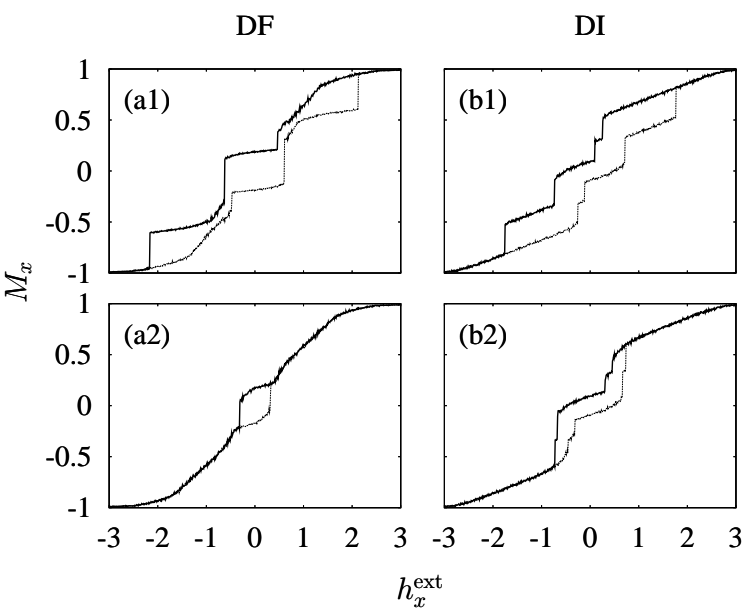

Fig. 3. The hysteresis loops for $T=0.01 T_{0}$ revealed by SOM network for DF and DI array. The boldface branches belong to $\Delta h^{\text {ext }}<0$.

By analyzing the revealed loops we conclude that two massive magnetization jumps contribute to the reversal. Deeper insight to the mechanism of their nucleation is offered by the configuration of moments. The sequence of snapshots that correspond to $\Delta h_{\mathrm{x}}^{\mathrm{ext}}<0$ regime is shown in Fig.4. We see that the defect evidently supports the nucleation of the intermediate inter-dot leaf. Without defect the noncolinear antiferromagnetic order is prefered.

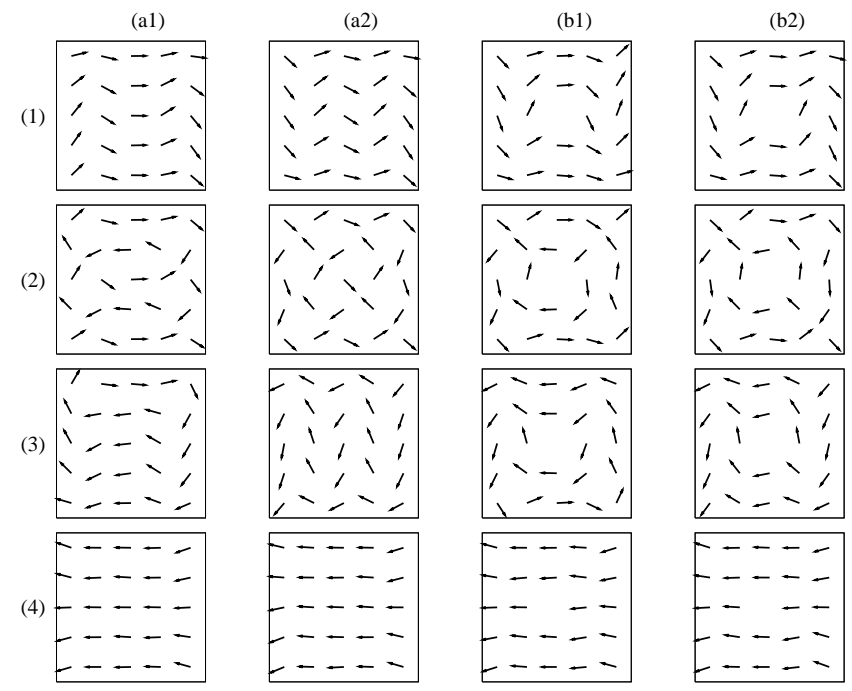

Fig. 4. The configuration of magnetic moments along the hysteresis path that belongs to representative loop types obtained for decreasing $h_{\mathrm{x}}^{\text {ext }}$ equal to (1) $1.5,(2) 0.0,(3)-1.0,(4)-3.0$. 


\section{Relaxation process}

The additional principle of defect detection is provided by relaxation process in zero external field starting from $M_{\mathrm{x}}=1$ state. The relaxation curves for DF and DI systems are plotted in Fig.5. For $T=0.01 T_{0}$ the qualitative differences in the relaxation of magnetization become clear. The most anomalous aspect of relaxation of DI is the pronounced peak. However, DF MDA does not exhibit this feature. The configurations show that in DF case the pair of antiparallel vortices is formed in contrast to inter-dot flower observed in DI case. As for the hysteresis, when the energy barriers are over bridged by thermal fluctuations, the transition to relaxation mode without peak is expected. This scenario is confirmed by the inset of Fig. 5, where the nearly exponential relaxation occurs without substantial marks of defect sensitivity.

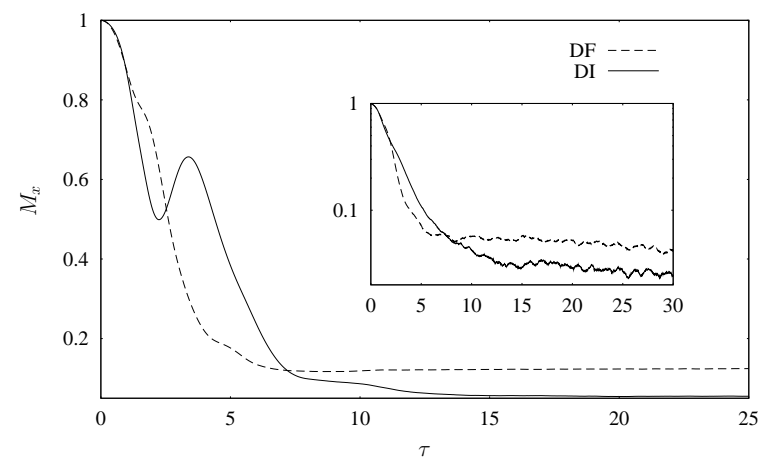

Fig. 5. The averaged magnetization relaxation obtained for DF and DI systems at temperatures $T=0.01 T_{0}$. Inset corresponds to $T=0.5 T_{0}$. The final curves are averages obtained from 1000 stochastic relaxation events.

\section{Conclusion and discussion}

The simulation statistical study discusses the theoretical grounds for defect detection in MDA. It has been shown, that that the presence of non-magnetic central node defect in a a small segment of MDA has non-negligible influence on dynamical properties of MDA, concretely magnetic hysteresis and relaxation. Further, it shows that the including the thermal activation is not only the additional factor of realistic simulation, but also the factor that can substantially enhance the chance to identify defect.

Finally, we are concluding with the believe that our present results will affect the experimental claims in this direction.

The authors would like to thank for financial support through grants VEGA 1/2009/05, APVT-51-052702, APVV-LPP-0030-06.
[1] J. I. Martín, J. Nogués, K. Liu, J. Vicent, I. K. Schuller: J. Magn. Magn. Matter. 256, 449 (2003).

[2] G. Srajer et al.: J. Magn. Magn. Matter. 307, 1-31 (2006).

[3] C. A. Ross et al.: Phys. Rev. B 65, 144417 (2002).

[4] D. Horváth, M. Gmitra, P. Baláž: Czech. J. Phys. 54, Suppl. D, 117 (2004).

[5] D. Horváth, M. Gmitra, P. Baláž: Phys. Stat. Sol. (b) 241, No. 15, 3624-3635 (2004).

[6] J. L. Duvail, S. Dubois and L. Piraux: J. Appl. Phys. 846352 (1998).

[7] C. Thirion, W. Wernsdorfer, and D. Mailly: Nat. Mater. 2, 524 (2003).

[8] W. K. Hiebert, A. Stankiewicz, and M. R. Freeman: Phys. Rev. Lett. 79, 1134 (1997).

[9] G. Prinz and K. Gathaway (Eds.): Physics Today, AIP, New York, 24 (1995).

[10] S. Y. Chou: Proc. IEEE 85, 652 (1997).

[11] G. M. McCleeland, M. W. Hart, C. T. Rettner, M. E. Best, K. R. Carter and B. D. Terris: Appl. Phys. Lett. 81, 1483 (2002).

[12] C. A. Ross: Annu. Rev. Mater. Res. 31, 203 (2001).

[13] L. J. Heyderman, H. H. Solak, C. David, D. Atkinson, R. P. Cowburn, F. Nolting: Appl. Phys. Lett. 85, 4989 (2004).

[14] M. Albrecht, M. Hu, A, Moser, O. Hellwig, B. D. Terris: J. Appl. Phys. 97, 103910 (2005).

[15] M. A. Kayali, W. M. Saslow: Phys. Rev. B 70, 174404 (2004).

[16] R. L. Stamps, R. E. Camley: J. Magn. Magn. Matter 177-181, 813 (1998).

[17] R. L. Stamps, R. E. Camley: Phys. Rew. B 60, 11694 (1999).

[18] R. L. Stamps, R. E. Camley: Phys. Rew. B 60, 12264 (1999).

[19] L. F. Zhang, C. Xu, P. M. Hui, Y. Q. Ma: J. Appl. Phys. 97, 103912 (2005).

[20] P. Majchrák, D. Horváth, M. Gmitra, I. Vávra: Proc. SPIE 5445 (2003) 262.

[21] D. Horváth, M. Gmitra, P. Majchrák, P. Baláž, I. Vávra: J. Magn. Magn. Matter 304, 486 (2006).

[22] W. Scholz, W. Schrefl, J. Fidler: J. Magn. Magn. Matter 233, 296 (2001).

[23] J. L. García-Palacios, F. J. Lázaro: Phys. Rew. B 58, 14937 (1998).

[24] P. C. Fannin, C. N. Marin: J. Magn. Magn. Matter 299, 425 (2006).

[25] Z. Li, S. Zhang: Phys. Rew. B 68, 024404 (2003).

[26] S. Haykin, "Neural networks" by Prentice Hall. Inc. Simon and Schuster/A Viacom Company Upper-Saddle River, New Jersey 07458, 1999.

[27] M. Gmitra, D. Horváth, D. Reitzner: J. Czech. Phys., 54 D631 (2004). 